\title{
Elliptic equations in the plane satisfying a Carleson measure condition
}

\section{Martin Dindoš and David J. Rule}

\begin{abstract}
In this paper we settle (in dimension $n=2$ ) the open question whether for a divergence form equation $\operatorname{div}(A \nabla u)=0$ with coefficients satisfying certain minimal smoothness assumption (a Carleson measure condition), the $L^{p}$ Neumann and Dirichlet regularity problems are solvable for some values of $p \in(1, \infty)$. The related question for the $L^{p}$ Dirichlet problem was settled (in any dimension) in 2001 by Kenig and Pipher [11].
\end{abstract}

\section{Introduction}

In this paper we prove some two dimensional results for second order elliptic operators under certain natural, minimal conditions on the coefficients of these operators. Primarily, our operators $L$ are of divergence form $L u=$ $\operatorname{div}(A \nabla u)$, where $A(X)=\left(a_{i j}(X)\right)$ is a (not necessarily symmetric) strongly elliptic matrix in the sense that there exists a positive constant $\lambda$ such that

$$
\lambda|\xi|^{2} \leq \sum_{i j} a_{i j}(X) \xi_{i} \xi_{j} \leq \lambda^{-1}|\xi|^{2}
$$

for all $X$ and $\xi \in \mathbb{R}^{2}$.

The motivation for the conditions placed upon the matrix $A$ come from the following example due to Kenig and Pipher [11] who studied the $L^{p}$ Dirichlet boundary value problem for elliptic equations in divergence form: $\operatorname{div}(A \nabla u)=0$. Consider the Dirichlet problem for the Laplacian $\Delta=\partial_{x}^{2}+\partial_{t}^{2}$.

$$
\begin{cases}\Delta u=0, & \text { in } \Omega_{\phi} \\ u=f_{0}, & \text { on } \partial \Omega_{\phi}\end{cases}
$$

2000 Mathematics Subject Classification: Primary 35J25; Secondary 35J67.

Keywords: elliptic equations, Carleson measure condition, Neumann problem, regularity problem, distributional inequalities, inhomogeneous equation. 
where $\Omega_{\phi}=\{(x, t) \mid \phi(x)<t\}$ and $\phi: \mathbb{R} \rightarrow \mathbb{R}$ is a Lipschitz function. It is well-know [2] that the $L^{2}$ Dirichlet boundary value problem is solvable for such a domain and operator, that is, we have the following estimate for the nontangential maximal function $N_{\phi, a}$ (see below for the definition):

$$
\left\|N_{\phi, a}(u)\right\|_{L^{2}(\mathbb{R})} \leqslant C\left\|f_{0}\right\|_{L^{2}\left(\partial \Omega_{\phi}\right)} .
$$

We now consider the transformation $\Phi: \mathbb{R}_{+}^{2} \rightarrow \Omega_{\phi}$, used by Dahlberg, Kenig and Stein (see [3] and [5]), but also earlier by Nečas [13], defined as

$$
\Phi(x, t)=\left(x, c_{0} t+\left(\theta_{t} * \phi\right)(x)\right),
$$

where $\left\{\theta_{t}\right\}_{t>0}$ is a smooth compactly supported approximate identity and $c_{0}$ can be chosen large enough, depending only on $\left\|\phi^{\prime}\right\|_{L^{\infty}(\mathbb{R})}$, so that $\Phi$ is oneto-one. One may compute that the function $\Phi$ enjoys the properties

- $|\partial \Phi(x, t)| \leqslant C$,

- $\left|\partial^{2} \Phi(x, t)\right| \leqslant C / t$, and

- $t\left|\partial^{2} \Phi(x, t)\right|^{2} d x d t$ is a Carleson measure.

Moreover, it is straightforward to see that the composition $w=u \circ \Phi$ is such that $\operatorname{div}(A \nabla w)=0$ in $\mathbb{R}_{+}^{2}$, where $A=(\operatorname{det} \Phi)\left(\left(\Phi^{\prime}\right)^{-1}\right)^{t}\left(\Phi^{\prime}\right)^{-1}$. Therefore $A$ inherits from $\Phi$ the properties

(i) $|\partial A(x, t)| \leqslant c_{1} / t$ and

(ii) $t|\partial A(x, t)|^{2} d x d t$ is a Carleson measure with norm $c_{1}$,

and from (1.1) we can see the corresponding non-tangential estimate

$$
\left\|N_{0, a}(w)\right\|_{L^{2}(\mathbb{R})} \leqslant C\left\|f_{0}(\cdot, \phi(\cdot))\right\|_{L^{2}(\mathbb{R})}
$$

holds. So the natural question is: "Are the conditions (i) and (ii) sufficient to conclude estimate (1.3) for an arbitrary solution to a divergence form equation?" Kenig and Pipher [11] prove an $L^{p}$ version of such estimate holds under closely related conditions, where $p>1$ may possibly be large and Dindoš, Petermichl and Pipher [6] show that the $L^{p}$ version holds for any given $1<p<\infty$ under the same assumptions, but when $c_{1}$ is sufficiently small (depending on $p$ ). In fact, both of these results are proved for bounded Lipschitz domains in any dimension and the elliptic equation may have lowerorder drift terms satisfying a similar Carleson measure condition.

Given [9], the same motivation can be used to justify posing the same question regarding the regularity and Neumann problems. We will obtain an analogous result to that proved for the Dirichlet problem, more specifically, for a given $p \in(1,2]$, we prove that the regularity problem $(R)_{p}$ and the Neumann problem $(N)_{p}$ hold under the hypotheses (i) and (ii) provided $c_{1}$ is sufficiently small. 
We remark that our method does not allow one to move beyond dimension two, that is, this problem is completely open for $n \geq 3$.

We note here that we eventually want to replace the conditions (i) and (ii) by an averaging condition on coefficients of $A$ as was done in [6] for the Dirichlet problem. In this paper, a solvability result was first established with a condition similar to (i) and (ii). Then this condition was replaced by a weaker averaging condition by the use of perturbation theory that is known for the Dirichlet problem. Moreover, our $\widetilde{N}_{\phi, a, \alpha}$ defined below is a slightly weaker $L^{1}$ average of the $L^{2}$ average used elsewhere. What we present here should therefore be considered as a first step on the road that will bring our knowledge of the Neumann and regularity problem to the same level as the Dirichlet problem.

The layout of this paper is as follows. In Section 2 we introduce some notation, state the main result, Theorem 2.2, and provide an outline of its proof. In Section 3 we reduce the proof of Theorem 2.2 to several inequalities, which are then proved.

\section{The Main Result}

First we fix some well-known notation adapted to the domain $\Omega_{\phi}$, where $\phi$ is a Lipschitz function. The $p$-adapted square function $S_{p, \phi, a}(g)$ with aperture $a$ of a function $g: \Omega_{\phi} \rightarrow \mathbb{R}$ is defined by

$$
S_{p, \phi, a}(g)(y)=\left(\iint_{\Gamma_{a}(y, \phi(y))}|\nabla g(x, t)|^{2}|g(x, t)|^{p-2} d x d t\right)^{\frac{1}{p}},
$$

where $\Gamma_{a}(y, \phi(y))=\{(x, t)|| x-y \mid \leqslant a(t-\phi(y))\}$ and we take $a<\left\|\phi^{\prime}\right\|_{L^{\infty}(\mathbb{R})}^{-1}$. We define the non-tangential maximal function of $g: \Omega_{\phi} \rightarrow \mathbb{R}$ as

$$
N_{\phi, a}(g)(y)=\sup _{\Gamma_{a}(y, \phi(y))}|g|
$$

and, for $g: \mathbb{R}_{+}^{2} \rightarrow \mathbb{R}$ and $\alpha \in(0,1 / 2]$,

$$
m_{\alpha}(g)(x, t)=\frac{1}{\left|B_{\alpha t}(x, t)\right|} \iint_{B_{\alpha t}(x, t)}|g|,
$$

where $B_{r}(x, t)=\left\{(y, s) \mid(y-x)^{2}+(s-t)^{2} \leqslant r^{2}\right\}$ is a ball with radius $r$ and centre $(x, t)$. Finally, we set $\widetilde{N}_{\phi, a, \alpha}(g)=N_{\phi, a}\left(m_{\alpha}(g)\right)$.

We will consider the Dirichlet problem

$$
\begin{cases}L u=0, & \text { in } \mathbb{R}_{+}^{2} \\ u=f_{0}, & \text { on } \partial \mathbb{R}_{+}^{2}\end{cases}
$$


with data $f_{0}: \partial \mathbb{R}_{+}^{2} \rightarrow \mathbb{R}$ and the Neumann problem

$$
\begin{cases}L u=0, & \text { in } \mathbb{R}_{+}^{2} \\ \nu \cdot A \nabla u=g_{0}, & \text { on } \partial \mathbb{R}_{+}^{2}\end{cases}
$$

with data $g_{0}: \partial \mathbb{R}_{+}^{2} \rightarrow \mathbb{R}$. The operator $L=\operatorname{div} A \nabla$ is an elliptic operator in divergence form with coefficients given by the matrix $A=\left(a_{i j}\right)_{i j}$. The matrix $A$ is assumed to have real-valued bounded measurable entries $\left(\max _{i, j}\left\|a_{i j}\right\|_{L^{\infty}(\Omega)}=\Lambda<\infty\right)$ and satisfy the uniform ellipticity condition

$$
\lambda|\xi|^{2} \leqslant \xi \cdot A \xi
$$

for some $\lambda>0$ and all $\xi \in \mathbb{R}^{2}$, but $A$ is not necessarily symmetric. In addition we will assume

$$
(x, t) \mapsto \sum_{i j}\left|\nabla a_{i j}\right|^{2} t d x d t
$$

is a Carleson measure with norm no more than $c_{1}$, that is to say

$$
\sup _{I} \frac{1}{|I|} \iint_{\widehat{I}} \sum_{i j}\left|\nabla a_{i j}\right|^{2} t d x d t \leqslant c_{1},
$$

where $\widehat{I}=I \times(0,|I|) \in \mathbb{R}^{2}$. Finally, we assume $\sup _{i j}\left|\nabla a_{i j}(x, t)\right| \leqslant c_{1} / t$ for all $(x, t) \in \mathbb{R}_{+}^{2}$. Both (2.1) and (2.2) have weak solutions for data in the set $\dot{C}_{0}^{\infty}$ of compactly supported smooth functions with mean-value zero. Such functions are dense in $L^{p}\left(\partial \mathbb{R}_{+}^{2}\right)$ for $1<p<\infty$. (See [12].)

Definition 2.1 (i) We say that the Dirichlet problem holds for $p$, or $(D)_{p}^{A}$ $=(D)_{p}$ holds, if for any $u$ solving (2.1) with boundary data $f_{0} \in L^{p}\left(\partial \mathbb{R}_{+}^{2}\right) \cap$ $\dot{C}_{0}^{\infty}\left(\partial \mathbb{R}_{+}^{2}\right)$ we have

$$
\left\|N_{0, a}(u)\right\|_{L^{p}\left(\partial \mathbb{R}_{+}^{2}\right)} \leqslant C(p)\left\|f_{0}\right\|_{L^{p}\left(\partial \mathbb{R}_{+}^{2}\right)} .
$$

(ii) We say that the Neumann problem holds for $p$, or $(N)_{p}^{A}=(N)_{p}$ holds, if for any $u$ solving (2.2) with boundary data $g_{0} \in L^{p}\left(\partial \mathbb{R}_{+}^{2}\right) \cap \dot{C}_{0}^{\infty}\left(\partial \mathbb{R}_{+}^{2}\right)$ we have

$$
\left\|\widetilde{N}_{0, a, \alpha}(\nabla u)\right\|_{L^{p}\left(\partial \mathbb{R}_{+}^{2}\right)} \leqslant C(p)\left\|g_{0}\right\|_{L^{p}\left(\partial \mathbb{R}_{+}^{2}\right)} .
$$

(iii) We say that the regularity problem holds for $p$, or $(R)_{p}^{A}=(R)_{p}$ holds, if for any $u$ solving (2.1) with boundary data $f_{0} \in W^{1, p}\left(\partial \mathbb{R}_{+}^{2}\right) \cap \dot{C}_{0}^{\infty}\left(\partial \mathbb{R}_{+}^{2}\right)$ we have

$$
\left\|\widetilde{N}_{0, a, \alpha}(\nabla u)\right\|_{L^{p}\left(\partial \mathbb{R}_{+}^{2}\right)} \leqslant C(p)\left\|\partial_{\tau} f_{0}\right\|_{L^{p}\left(\partial \mathbb{R}_{+}^{2}\right)} .
$$

In each case, the constant $C(p)>0$ must depend only on $\lambda, \Lambda, \Omega, a, \alpha$ and $p$. 
Our main result is the following.

Theorem 2.2 Let $L=\operatorname{div}(A \nabla \cdot)$ be an elliptic operator as described above. For each fixed $1<p \leqslant 2$, there exist $a, \alpha$ and $c_{2}$ such that, if $c_{1}<c_{2}$, then $(R)_{p}^{A}$ and $(N)_{p}^{\widetilde{A}}$ hold, where $\widetilde{A}=A^{t} / \operatorname{det}(A)$.

The proof of Theorem 2.2 will be based on a duality estimate. Consider the inhomogeneous Dirichlet problem

$$
\begin{cases}\operatorname{div}\left(A^{t} \nabla v\right)=\operatorname{div}(F) & \text { in } \mathbb{R}_{+}^{2}, \\ v=0, & \text { on } \partial \mathbb{R}_{+}^{2}\end{cases}
$$

We compute

$$
\begin{aligned}
\iint_{\mathbb{R}_{+}^{2}}(\nabla u) F d x d t & =-\iint_{\mathbb{R}_{+}^{2}} u \operatorname{div}(F) d x d t+\int_{\partial \mathbb{R}_{+}^{2}} u \nu \cdot F d x \\
& =-\iint_{\mathbb{R}_{+}^{2}} u \operatorname{div}\left(A^{t} \nabla v\right) d x d t+\int_{\partial \mathbb{R}_{+}^{2}} u \nu \cdot F d x \\
& =-\iint_{\mathbb{R}_{+}^{2}} \operatorname{div}(A \nabla u) v d x d t+\int_{\partial \mathbb{R}_{+}^{2}} u \nu \cdot\left(F-A^{t} \nabla v\right) d x \\
& =\int_{\partial \mathbb{R}_{+}^{2}} u \nu \cdot\left(F-A^{t} \nabla v\right) d x
\end{aligned}
$$

Now, for a solution $v$ to $(2.4)$, we can define a conjugate $\bar{v}$ to $v$ via the system

$$
A^{t} \nabla v-F=\left(\begin{array}{cc}
0 & 1 \\
-1 & 0
\end{array}\right) \nabla \bar{v}
$$

This is only possible because we are in two dimensions. Importantly, it allows us to write

$$
\begin{aligned}
\iint_{\mathbb{R}_{+}^{2}}(\nabla u) F d x d t & =\int_{\partial \mathbb{R}_{+}^{2}} u \nu \cdot\left(F-A^{t} \nabla v\right) d x \\
& =-\int_{\partial \mathbb{R}_{+}^{2}} u \partial_{\tau} \bar{v} d x=\int_{\partial \mathbb{R}_{+}^{2}} \partial_{\tau} u \bar{v} d x
\end{aligned}
$$

From here we can easily see how we might obtain our main result. The idea is that we can now test $\nabla u$ against a suitable class of dual functions $F$, with norm $\|F\|_{[p]}$, say, via the above equality. We can conclude

$$
\left\|\tilde{N}_{0, a, \alpha}(\nabla u)\right\|_{L^{p}\left(\partial \mathbb{R}_{+}^{2}\right)} \leqslant C\left\|\partial_{\tau} f_{0}\right\|_{L^{p}\left(\partial \mathbb{R}_{+}^{2}\right)},
$$

provided $\|\bar{v}\|_{L^{p}\left(\partial \mathbb{R}_{+}^{2}\right)} \leqslant C\|F\|_{[p]}$. This would prove that $(R)_{p}^{A}$ holds; that $(N)_{p}^{\widetilde{A}}$ holds would then follow by considering a conjugate for a solution to (2.1) defined similarly to (2.5) (see [12]). 
We now need to describe an appropriate class of $F$, but first we will fix some well-known notation. For a Lipschitz function $\phi, \Omega=\Omega_{\phi}:=$ $\{(x, t) \mid t>\phi(x)\}$. For a measure $f$ on $\Omega_{\phi}$, we define

$$
C_{\phi}(f)(y)=\sup _{I \ni y}\left(\frac{1}{|I|} \iint_{\widehat{I}_{\phi}}|d f|\right)
$$

where $\widehat{I}_{\phi}=\{(x, t)|x \in I, \phi(x)<t<\phi(x)+| I \mid\}$ is a tent over the interval $I$. When we write $C_{\phi}(F)$, where $F$ is is a function on $\Omega_{\phi}$, we mean the natural modification

$$
C_{\phi}(F)(y)=\sup _{I \ni y}\left(\frac{1}{|I|} \iint_{\widehat{I}_{\phi}}|F(x, t)| d x d t\right) .
$$

We know from [1] that

$$
\iint_{\Omega_{\phi}} G d f \leqslant C\left(\left\|\phi^{\prime}\right\|_{L^{\infty}(\mathbb{R})}, a\right) \int N_{\phi, a}(G)(y) C_{\phi}(f)(y) d y
$$

and the space of measures $f$ such that $C_{\phi}(f) \in L^{p}(\mathbb{R})$ is the dual of the space of functions $G$ such that $N_{\phi, a}(G) \in L^{p^{\prime}}(\mathbb{R})$. We will also need the inequality from [1]

$$
\begin{aligned}
& \iint_{\Omega_{\phi}} F(x, t) \nabla G(x, t) t d x d t \leqslant \\
& \quad \leqslant C\left(\left\|\phi^{\prime}\right\|_{L^{\infty}(\mathbb{R})}, a\right) \int C_{\phi}\left(t F^{2}\right)(y)^{\frac{1}{2}} S_{2, \phi, a}(G)(y) d y .
\end{aligned}
$$

Let $\varphi \in C^{\infty}\left(\mathbb{R}^{2}\right)$ be a fixed positive function, having support in $B_{5 / 6}(0)$ and such that $\varphi \equiv 1$ on $B_{4 / 6}(0)$. Then $\psi=\varphi_{\alpha / 12} * \varphi_{\alpha}$, where, for $\alpha>0$, $\varphi_{\alpha}=\alpha^{-2} \varphi\left(\alpha^{-1} \cdot\right)$, has support in $B_{\alpha}(0)$ and $\psi \equiv 1$ on $B_{\alpha / 2}(0)$. We then consider datum $F$ in (2.4) of the form

$$
F(X)=\chi(X)\left(\psi_{t} * f\right)(X)=\chi(X)\left(\varphi_{\alpha t / 12} * \varphi_{\alpha t} * f\right)(X)
$$

where $X=(x, t),|\chi| \leqslant 1$ and $f$ is a measure such that $C_{0}(f) \in L^{p}\left(\partial \mathbb{R}_{+}^{2}\right)$ (i.e. we use $C_{\phi}(f)$ defined above for the function $\phi=0$ ). The norm placed on the set of such $F$ is then

$$
\|F\|_{[p]}=\left\|C_{0}(f)\right\|_{L^{p}(\mathbb{R})} .
$$

With $F$ as in (2.8), by Fubini's Theorem we have

$$
\iint_{\mathbb{R}_{+}^{2}} F(x, t) G(x, t) d x d t=\iint_{\mathbb{R}_{+}^{2}} \iint_{\mathbb{R}_{+}^{2}} \psi_{t}(X-Y) \chi(X) G(X) d X d f(Y)
$$


where $X=(x, t)$. An straightforward calculation shows

$$
m_{\alpha / 4}(G)(Y) \leqslant \iint_{\mathbb{R}_{+}^{2}} \psi_{t}(X-Y) \chi(X) G(X) d X \leqslant C m_{2 \alpha}(G)(Y)
$$

for $\alpha \leqslant 1 / 2$ and $\chi(X)=G(x) /|G(x)|$, so with the duality above, the set of $F$ as in (2.8) with norm $\left\|C_{0}(f)\right\|_{L^{p}(\mathbb{R})}$ is an appropriate set of $F$. To complete the proof it suffices to prove for $2 \leqslant p<\infty$ the inequality

$$
\left\|N_{0, a}(\bar{v})\right\|_{L^{p}(\mathbb{R})} \leqslant C\left\|C_{0}(f)\right\|_{L^{p}(\mathbb{R})}
$$

under the hypothesis $c_{1}$ is sufficiently small. We will do this in Section 3 .

It would be interesting to also prove (2.10) for $1<p<2$ when $c_{1}$ is small as this would imply the regularity and Neumann problems held for any given $p$ when $c_{1}$ is sufficiently small, as is the case for the Dirichlet problem [6]. Unfortunately we are not able to do this. Possibly an even more interesting question would be whether or not the Neumann and regularity problems can be shown to hold for some (small) exponent $p$ when $c_{1}$ is only assumed to be finite. Given [12] and [11] one would conjecture this is the case, but again our methods are not powerful enough to do this. To prove this conjecture via the methods used here, one would require better knowledge of the constants involved our estimates.

\section{Estimates for the Inhomogeneous Equation}

It will be useful to consider an inhomogeneous Dirichlet problem which is slightly more general than (2.4):

$$
\begin{cases}L^{t} v=\operatorname{div}(F), & \text { in } \mathbb{R}_{+}^{2} \\ v=f_{0}, & \text { on } \partial \mathbb{R}_{+}^{2}\end{cases}
$$

with data $f_{0}: \partial \mathbb{R}_{+}^{2} \rightarrow \mathbb{R}$ and $F: \mathbb{R}_{+}^{2} \rightarrow \mathbb{R}^{2}$. We make the same assumptions on $L$ here as we did in (2.1) and (2.2) (and $L^{t}=\operatorname{div}\left(A^{t} \nabla \cdot\right)$ ).

With $F$ as in (2.8), by Fubini's Theorem and (2.6) we have

$$
\begin{aligned}
\iint_{\Omega_{\phi}} F(x, t) G(x, t) d x d t & \leqslant \iint_{\Omega_{c_{3} \phi}}\left|\left(\varphi_{\alpha t} * f\right)(y, s)\right| m_{\alpha / 10}(G)(y, s) d y d s \\
& \leqslant C \int C_{c_{3} \phi}\left(m_{\alpha}(f)\right) N_{c_{3} \phi, a}\left(m_{\alpha / 10}(G)\right) d y
\end{aligned}
$$

where $c_{3}=1-\alpha \sqrt{1+\left\|\phi^{\prime}\right\|_{\infty}^{2}} / 10$. We will use this later. 
Observe that we are free to assume $A$ is upper triangular, provided we introduce lower order terms. Indeed

$$
L v=\partial_{x}\left(a_{11} \partial_{x} v+\left(a_{12}+a_{21}\right) \partial_{t} v\right)+\partial_{t}\left(a_{22} \partial_{t} v\right)+\left(\partial_{t} a_{21}\right) \partial_{x} v-\left(\partial_{x} a_{21}\right) \partial_{t} v .
$$

In this section we set about proving that solutions $v$ to (3.1) satisfy the estimates

$$
\left\|N_{0, a}(v)\right\|_{L^{l}\left(\partial \mathbb{R}_{+}^{2}\right)} \leqslant C(p, l)\left(\left\|S_{p, 0, a}(v)\right\|_{L^{l}\left(\partial \mathbb{R}_{+}^{2}\right)}+\left\|C_{0}(f)\right\|_{L^{l}\left(\partial \mathbb{R}_{+}^{2}\right)}\right)
$$

for $2 \leqslant p<\infty$ and $0<l<\infty$ and

$$
\left\|S_{p, 0, a}(v)\right\|_{L^{q}\left(\partial \mathbb{R}_{+}^{2}\right)} \leqslant C(p, q)\left(\left\|N_{0, a}(v)\right\|_{L^{q}\left(\partial \mathbb{R}_{+}^{2}\right)}+\left\|C_{0}(f)\right\|_{L^{q}\left(\partial \mathbb{R}_{+}^{2}\right)}\right)
$$

for $2 \leqslant p<\infty$ and $p<q<\infty$. These estimates can be viewed as a generalisation of the main estimates in [10], which themselves are closely related to the work of Dahlberg [4]. The methods we use are essentially the distributional inequality methods used in [10]. The estimate (3.3) is eventually achieved in Theorem 3.6. Then we move on to prove (3.4) which we state as Corollary 3.9. Lemma 3.11 enables us to relate the conjugate $\bar{v}$ to $v$ via the following estimate:

$$
\left\|S_{2,0, a}(\bar{v})\right\|_{L^{p}\left(\partial \mathbb{R}_{+}^{2}\right)} \leqslant C(p)\left(\left\|S_{2,0, a}(v)\right\|_{L^{p}\left(\partial \mathbb{R}_{+}^{2}\right)}+\left\|C_{0}(f)\right\|_{L^{p}\left(\partial \mathbb{R}_{+}^{2}\right)}\right)
$$

for $1<p<\infty$. The final ingredient required to prove (2.10) is the estimate

$$
\left\|S_{p, 0, a}(v)\right\|_{L^{p}\left(\partial \mathbb{R}_{+}^{2}\right)} \leqslant C(p)\left\|C_{0}(f)\right\|_{L^{p}\left(\partial \mathbb{R}_{+}^{2}\right)}
$$

which requires that $v$ have zero boundary value and $c_{1}$ be sufficiently small. The estimate (3.6) is proved in Lemma 3.10. Let us now take these estimates as given and use them to complete the proof of Theorem 2.2.

Proof of Theorem 2.2. As discussed in Section 2, it suffices to prove (2.10). We remark that $\bar{v}$ also satisfies an equation of the form (3.1) were the coefficient matrix has ellipticity constants which are multiples of those for $A$ and its first derivatives satisfy the same Carleson measure condition with constant being a multiple of $c_{1}$. Specifically, $A^{t}$ is replaced by $A / \operatorname{det}(A)$ and on the right-hand side, $F$ becomes a bounded matrix multiplied by $F$, and so is still of the form (2.8). This means that (3.3) and (3.4) are also valid for $\bar{v}$.

Now, by (3.3) and (3.5), we have

$$
\begin{aligned}
\left\|N_{0, a}(\bar{v})\right\|_{L^{p}\left(\partial \mathbb{R}_{+}^{2}\right)} & \leqslant C\left(\left\|S_{2,0, a}(\bar{v})\right\|_{L^{p}\left(\partial \mathbb{R}_{+}^{2}\right)}+\left\|C_{0}(f)\right\|_{L^{p}\left(\partial \mathbb{R}_{+}^{2}\right)}\right) \\
& \leqslant C\left(\left\|S_{2,0, a}(v)\right\|_{L^{p}\left(\partial \mathbb{R}_{+}^{2}\right)}+\left\|C_{0}(f)\right\|_{L^{p}\left(\partial \mathbb{R}_{+}^{2}\right)}\right) .
\end{aligned}
$$

When $p=2$ we can use (3.6) to obtain the bound $C\left\|C_{0}(f)\right\|_{L^{2}\left(\partial \mathbb{R}_{+}^{2}\right)}$, as required. 
When $p>2$ we may first use (3.4) and then (3.3) and (3.6) to obtain once again

$$
\begin{aligned}
\left\|N_{0, a}(\bar{v})\right\|_{L^{p}\left(\partial \mathbb{R}_{+}^{2}\right)} & \leqslant C\left(\left\|N_{0, a}(v)\right\|_{L^{p}\left(\partial \mathbb{R}_{+}^{2}\right)}+\left\|C_{0}(f)\right\|_{L^{p}\left(\partial \mathbb{R}_{+}^{2}\right)}\right) \\
& \leqslant C\left(\left\|S_{p, 0, a}(v)\right\|_{L^{p}\left(\partial \mathbb{R}_{+}^{2}\right)}+\left\|C_{0}(f)\right\|_{L^{p}\left(\partial \mathbb{R}_{+}^{2}\right)}\right) \\
& \leqslant C\left\|C_{0}(f)\right\|_{L^{p}\left(\partial \mathbb{R}_{+}^{2}\right)} .
\end{aligned}
$$

Thus, we are left with the task of establishing (3.3), (3.4), (3.5) and (3.6). We remark that it is sufficient to establish these inequalities only for exponents $p=2^{k}$, where $k \in \mathbb{N}$. This establishes the boundedness of $\partial_{\tau} f_{0} \mapsto \widetilde{N}_{0, a, \alpha}(\nabla u)$ on the $L^{p^{\prime}}\left(\partial \mathbb{R}_{+}^{2}\right)$ for $p^{\prime}=\left(1-2^{-k}\right)^{-1}$ and we can use interpolation to obtain all $p^{\prime} \in(1,2]$.

Lemma 3.1 Let $v$ be a solution to (3.1) as described above and let $\phi$ and $\phi_{+}$ be non-negative Lipschitz functions such that $\phi_{+} \geqslant 2 \phi$. Let I be an interval in $\mathbb{R}$ with $r:=|I|$ and $p=2^{k}$ for some $k \in \mathbb{N}$. Then there exists a sufficiently small choice of $a$ and $\alpha$, depending only on $\lambda, \Lambda, p, c_{1}$ and $\left\|\phi_{+}^{\prime}\right\|_{L^{\infty}(\mathbb{R})}$, and a constant $C(p)$, depending on the same parameters, along with a and $\alpha$, such that

$$
\begin{aligned}
\left\|v\left(\cdot, \phi_{+}(\cdot)\right)\right\|_{L^{p}(I)}^{p} \leqslant & C(p)\left(\left\|S_{p, \phi, a}(v)\right\|_{L^{p}(3 I)}^{p}+\left\|C_{\phi}\left(m_{\alpha}(f)\right)\right\|_{L^{p}(3 I)}^{p}\right. \\
& +\left\|N_{\phi, a}(v)\right\|_{L^{p}(3 I)}^{p-1}\left\|C_{\phi}\left(m_{\alpha}(f)\right)\right\|_{L^{p}(3 I)} \\
& +\left\|N_{\phi, a}(v)\right\|_{L^{p}(3 I)}^{(p-2) / 2}\left\|S_{p, \phi, a}(v)\right\|_{L^{p}(3 I)}^{p / 2}\left\|C_{\phi}\left(m_{\alpha}(f)\right)\right\|_{L^{p}(3 I)} \\
& \left.+\left\|N_{\phi, a}(v)\right\|_{L^{p}(3 I)}^{p / 2}\left\|S_{p, \phi, a}(v)\right\|_{L^{p}(3 I)}^{p / 2}+r\left|v\left(X_{r}\right)\right|^{p}\right),
\end{aligned}
$$

where $X_{r}$ is any point in $\left\{(x, t) \mid x \in 3 I, \phi_{+}(x)+r / 2 \leqslant t \leqslant \phi_{+}(x)+3 r / a\right\}$.

Proof. In order to facilitate the use of integration by parts, we will use the mapping from $\mathbb{R}_{+}^{2}$ to $\Omega_{\phi}$ defined by (1.2). This maps $v$ to a solution of an equation of the same form as (3.1), with data controlled by multiples of the constants controlling the data of the original equation. We will denote this new solution by $w=v \circ \Phi$.

We choose a smooth function $\xi_{1}: \mathbb{R} \rightarrow \mathbb{R}$ such that $\xi_{1}(x)=1$ for $x \in I$, $\left|\xi_{1}^{\prime}\right| \leqslant 5 / r$ and support contained in $2 I$. Choose a second $\xi_{2}:[0, \infty) \rightarrow \mathbb{R}$ such that $\xi_{2}(t)=1$ for $t \in[0, r],\left|\xi_{1}^{\prime}\right| \leqslant 5 / r$ and support contained in $[0,2 r]$. Now define $\xi(x, t)=\xi_{1}(x) \xi_{2}(t)$. of all,

We proceed by calculating in a similar fashion to [10], [11] and [14]. First

$$
\begin{aligned}
\int w(x, 0)^{p} \xi_{1}(x) d x & =-\iint_{\mathbb{R}_{+}^{2}} \partial_{t}\left(w^{p} \xi\right)(x, t) d x d t \\
& =-\iint_{\mathbb{R}_{+}^{2}} p w^{p-1} w_{t} \xi d x d t-\iint_{\mathbb{R}_{+}^{2}} w^{p} \xi_{1} \xi_{2}^{\prime} d x d t
\end{aligned}
$$


The second term on the right-hand side of (3.7) is controlled by

$$
r^{-1} \iint_{K} w^{p} d x d t
$$

where $K=\{(x, t) \mid x \in(7 / 3) I, r \leqslant t \leqslant 2 r\}$. Assume, for the moment,

$$
|w-c|^{p} \leqslant\left. C(p)|w| w\right|^{p-1}-c|c|^{p-1} \mid
$$

for all $w, c \in \mathbb{R}$ and $p \in \mathbb{N}$. Let $X_{r}$ be any point in $K$ and set

$$
K^{\prime}=\{(x, t) \mid x \in(8 / 3) I, 7 r / 8 \leqslant t \leqslant 17 r / 8\}
$$

We choose $c$ such that

$$
\left(w|w|^{p / 2-1}\right)_{K^{\prime}}=c|c|^{p / 2-1} \quad \text { where } \quad f_{K^{\prime}}=\frac{1}{\left|K^{\prime}\right|} \iint_{K^{\prime}} f .
$$

Using [7, Thm 8.17] and Poincaré's inequality, we may further estimate this term, using (3.8) with exponent $p / 2$,

$$
\begin{aligned}
& r^{-1} \iint_{K}\left(w-w\left(X_{r}\right)\right)^{p} d x d t+r^{-1} \iint_{K} w^{p}\left(X_{r}\right) d x d t \leqslant \\
& \leqslant C r \operatorname{osc}_{K}(w)^{p}+C r\left|w\left(X_{r}\right)\right|^{p} \\
& \leqslant C r \sup _{K}|w-c|^{p}+C r\left|w\left(X_{r}\right)\right|^{p} \\
& \leqslant C r^{-1} \iint_{K^{\prime}}|w-c|^{p} d x d t+C r^{1+p(1-2 / q)}\|F\|_{L^{q}(K)}^{p}+C r\left|w\left(X_{r}\right)\right|^{p} \\
& \leqslant\left. C r^{-1} \iint_{K^{\prime}}|w| w\right|^{p / 2-1}-\left.c|c|^{p / 2-1}\right|^{2} d x d t \\
& \quad \quad C r^{1+p(1-2 / q)}\|F\|_{L^{q}(K)}^{p}+C r\left|w\left(X_{r}\right)\right|^{p} \\
& \leqslant C r \iint_{K^{\prime}}\left|\nabla\left(w^{p / 2}\right)\right|^{2} d x d t+C r \inf _{x \in(8 / 3) I} C_{\phi}\left(m_{\alpha}(f)\right)(x)^{p}+C r\left|w\left(X_{r}\right)\right|^{p} \\
& \leqslant C\left\|S_{p, \phi, a}(v)\right\|_{L^{2}(3 I)}^{p}+\left\|C_{\phi}\left(m_{\alpha}(f)\right)\right\|_{L^{p}(3 I)}^{p}+C r\left|w\left(X_{r}\right)\right|^{p} .
\end{aligned}
$$

We now prove (3.8). This is done by considering several cases. First, if $w$ and $c$ are positive, and $w \geqslant c$, then there exists a constant $C(p)$ such that

$$
\begin{aligned}
& \left.|w| w\right|^{p-1}-c|c|^{p-1} \mid=w^{p}-c^{p}=(w-c) \sum_{k=0}^{p-1} w^{p-1-k} c^{k} \\
& \geqslant C(p)(w-c) \sum_{k=0}^{p-1}\left(\begin{array}{c}
p-1 \\
k
\end{array}\right) w^{p-1-k} c^{k}=C(p)(w-c)^{p}=C|w-c|^{p} .
\end{aligned}
$$


Secondly, if $w c \leqslant 0$, then

$$
\begin{aligned}
|w-c|^{p} & =(|w|+|c|)^{p} \leqslant 2^{p} \max \left(|w|^{p},|c|^{p}\right) \\
& \leqslant 2^{p}\left(|w|^{p}+|c|^{p}\right)=\left.2^{p}|w| w\right|^{p-1}-c|c|^{p-1} \mid .
\end{aligned}
$$

All other cases can easily be reduced to the first and so (3.8) is proved.

Returning to our main estimate, the first term on the right-hand side of (3.7) is

$$
\begin{array}{rl}
-\iint_{\mathbb{R}_{+}^{2}} & p w^{p-1} w_{t} \xi d x d t=-\iint_{\mathbb{R}_{+}^{2}} \frac{p w^{p-1} a_{22} w_{t} \xi}{a_{22}} \partial_{t}(t) d x d t \\
= & \iint_{\mathbb{R}_{+}^{2}} \partial_{t}\left(\frac{p w^{p-1} a_{22} w_{t} \xi}{a_{22}}\right) t d x d t \\
= & \iint_{\mathbb{R}_{+}^{2}}(p-1) w^{p-2} a_{22} w_{t}^{2} \xi \frac{p t d x d t}{a_{22}}+\iint_{\mathbb{R}_{+}^{2}} w^{p-1} \partial_{t}\left(a_{22} w_{t}\right) \xi \frac{p t d x d t}{a_{22}} \\
& +\iint_{\mathbb{R}_{+}^{2}} w^{p-1} a_{22} w_{t} \xi_{1} \xi_{2}^{\prime} \frac{p t d x d t}{a_{22}}-\iint_{\mathbb{R}_{+}^{2}} w^{p-1} w_{t} \xi \partial_{t} a_{22} \frac{p t d x d t}{\left(a_{22}\right)^{2}} \\
= & : \mathrm{I}+\mathrm{II}+\mathrm{III}+\mathrm{IV} .
\end{array}
$$

Using the fact that $w$ solves (3.1) (where, we recall, we may take $A$ to be upper triangular and introduce a lower order term, $B \cdot \nabla w)$ we see that II is equal to

$$
\begin{gathered}
\iint_{\mathbb{R}_{+}^{2}} w^{p-1} \operatorname{div}(F) \xi \frac{p t d x d t}{a_{22}}-\iint_{\mathbb{R}_{+}^{2}} w^{p-1} \partial_{x}\left(a_{11} w_{x}+a_{12} w_{t}\right) \xi \frac{p t d x d t}{a_{22}} \\
-\iint_{\mathbb{R}_{+}^{2}} w^{p-1} B \cdot \nabla w \xi \frac{p t d x d t}{a_{22}}=: \mathrm{II}_{1}+\mathrm{II}_{2}+\mathrm{II}_{3} .
\end{gathered}
$$

Integrating by parts we see that

$$
\begin{aligned}
\mathrm{II}_{1}=- & \iint_{\mathbb{R}_{+}^{2}}(p-1) w^{p-2} \nabla w \cdot F \xi \frac{p t d x d t}{a_{22}}+\iint_{\mathbb{R}_{+}^{2}} w^{p-1} F \cdot \nabla \xi \frac{p t d x d t}{a_{22}} \\
& +\iint_{\mathbb{R}_{+}^{2}} w^{p-1} \xi F \cdot\left(\begin{array}{c}
0 \\
1
\end{array}\right) \frac{p d x d t}{a_{22}}-\iint_{\mathbb{R}_{+}^{2}} w^{p-1} \xi F \cdot \nabla a_{22} \frac{p t d x d t}{\left(a_{22}\right)^{2}} .
\end{aligned}
$$

Transforming back to our original coordinate system via the inverse of (1.2), each of these terms can be controlled using (3.2). Indeed, using the facts that $\left|\nabla a_{22}(x, t)\right| \leqslant C c_{1} / t,|\nabla \xi| \leqslant 5 / r$ and that the integrands are zero 
when $t>2 r$ or $x \notin 2 I$, we see that

$$
\begin{aligned}
& \left|\iint_{\mathbb{R}_{+}^{2}}(p-1) w^{p-2} \nabla w \cdot F \xi \frac{p t d x d t}{a_{22}}\right| \\
& \leqslant C \int_{3 I} C_{c_{3} \phi_{+}}\left(m_{\alpha}(f)\right) N_{c_{3} \phi_{+}, a / 2}\left(m_{\alpha / 10}\left(t v^{p-2}|\nabla v|\right)\right) d x \\
& \left|\iint_{\mathbb{R}_{+}^{2}} w^{p-1} F \cdot \nabla \xi \frac{p t d x d t}{a_{22}}\right| \leqslant C \int_{3 I} C_{c_{3} \phi_{+}}\left(m_{\alpha}(f)\right) N_{c_{3} \phi_{+}, a / 2}\left(m_{\alpha / 10}\left(v^{p-1}\right)\right) d x \\
& \left|\iint_{\mathbb{R}_{+}^{2}} w^{p-1} \xi F \cdot\left(\begin{array}{c}
0 \\
1
\end{array}\right) \frac{p d x d t}{a_{22}}\right| \leqslant C \int_{3 I} C_{c_{3} \phi_{+}}\left(m_{\alpha}(f)\right) N_{c_{3} \phi_{+}, a / 2}\left(m_{\alpha / 10}\left(v^{p-1}\right)\right) d x
\end{aligned}
$$

and

$$
\left|\iint_{\mathbb{R}_{+}^{2}} w^{p-1} \xi F \cdot \nabla a_{22} \frac{p t d x d t}{a_{22}^{2}}\right| \leqslant C \int_{3 I} C_{c_{3} \phi_{+}}\left(m_{\alpha}(f)\right) N_{c_{3} \phi_{+}, a / 2}\left(m_{\alpha / 10}\left(v^{p-1}\right)\right) d x .
$$

So, using the simple observation that

$$
N_{c_{3} \phi_{+}, a / 2}\left(m_{\alpha / 10}\left(t v^{p-2}|\nabla v|\right)\right) \leqslant C N_{\phi, a}(v)^{(p-2) / 2} S_{p, \phi, a}(v)^{p / 2}
$$

for sufficiently small $a$ and $\alpha$, we find

$$
\begin{gathered}
\left|\mathrm{II}_{1}\right| \leqslant C\left(\left\|N_{\phi, a}(v)\right\|_{L^{p}(3 I)}^{(p-2) / 2}\left\|S_{p, \phi, a}(v)\right\|_{L^{p}(3 I)}^{p / 2}\left\|C_{\phi}\left(m_{\alpha}(f)\right)\right\|_{L^{p}(3 I)}\right. \\
\left.+\left\|N_{\phi, a}(v)\right\|_{L^{p}(3 I)}^{p-1}\left\|C_{\phi}\left(m_{\alpha}(f)\right)\right\|_{L^{p}(3 I)}\right) .
\end{gathered}
$$

Again, using integration by parts,

$$
\begin{aligned}
\mathrm{II}_{2}= & \iint_{\mathbb{R}_{+}^{2}}(p-1) w^{p-2} w_{x}\left(a_{11} w_{x}+a_{12} w_{t}\right) \xi \frac{p t d x d t}{a_{22}} \\
& +\iint_{\mathbb{R}_{+}^{2}} w^{p-1}\left(a_{11} w_{x}+a_{12} w_{t}\right) \partial_{x} \xi \frac{p t d x d t}{a_{22}} \\
& +\iint_{\mathbb{R}_{+}^{2}} w^{p-1}\left(a_{11} w_{x}+a_{12} w_{t}\right) \xi \partial_{x} a_{22} \frac{p t d x d t}{a_{22}^{2}}
\end{aligned}
$$

The first integral may be combined with I to produce

$$
\iint_{\mathbb{R}_{+}^{2}}(p-1) w^{p-2} \nabla w \cdot A \nabla w \xi \frac{p t d x d t}{a_{22}} \leqslant C\left\|S_{p, \phi, a}(v)\right\|_{L^{p}(3 I)}^{p} .
$$

What remains of $\mathrm{II}_{2}$ may be dealt with as above using (2.7) once we observe that, since $|\nabla \xi| \leqslant 5 / r$ and $\operatorname{supp}(\xi) \subset 2 I \times[0,2 r]$, we have $C_{0}\left(t|\nabla \xi|^{2}\right) \leqslant 100$. 
So, for example,

$$
\begin{gathered}
\left.\left|\iint_{\mathbb{R}_{+}^{2}}\right| w\right|^{p-1}|\nabla w||\nabla \xi| t d x d t \mid \leqslant C \int_{3 I} C_{0}\left(t|\nabla \xi|^{2}\right)(y)^{\frac{1}{2}} S_{2, \phi, a}\left(v^{p-1}|\nabla v|\right)(y) d y \\
\leqslant C \int_{3 I} N_{\phi, a}(v)^{p / 2} S_{p, \phi, a}(v)^{p / 2} d x . \leqslant C\left\|N_{\phi, a}(v)\right\|_{L^{p}(3 I)}^{p / 2}\left\|S_{p, \phi, a}(v)\right\|_{L^{p}(3 I)}^{p / 2}
\end{gathered}
$$

Thus we obtain

$$
\left|\mathrm{II}_{2}\right| \leqslant C\left(\left\|S_{p, \phi, a}(v)\right\|_{L^{p}(3 I)}^{p}+\left\|N_{\phi, a}(v)\right\|_{L^{p}(3 I)}^{p / 2}\left\|S_{p, \phi, a}(v)\right\|_{L^{p}(3 I)}^{p / 2}\right)
$$

The terms $\mathrm{II}_{3}$, III and IV can also be dealt with in this way, and so, combining all of these estimates, we obtain the lemma.

For any continuous function $v: \mathbb{R}_{+}^{2} \rightarrow \mathbb{R}$ and $\mu \in \mathbb{R}$, define

$$
h_{\phi, \mu, a}(v)(x)=\sup \left\{t \geqslant \phi(x) \mid \sup _{\Gamma_{a}(x, t)}(v)>\mu\right\} .
$$

Lemma 3.2 Provided $v$ is such that $h_{\phi, \mu, a}(v)<\infty$, the function $h_{\phi, \mu, a}(v)$ is Lipschitz with constant $1 / a$.

Proof. See, for example, [11, Lem 3.5].

Given a non-empty open proper subset $D \subset \mathbb{R}$ we can find a family of closed dyadic intervals $\left\{I_{j}\right\}_{j}$ such that:

(a) $\cup_{j} I_{j}=D$ and the $I_{j}$ have disjoint interiors;

(b) $\left|I_{j}\right| \leqslant \operatorname{dist}\left(I_{j}, D^{c}\right) \leqslant 4\left|I_{j}\right| ; \quad$ and

(c) If the boundaries of $I_{j}$ and $I_{k}$ touch then

$$
\frac{1}{4} \leqslant \frac{\left|I_{j}\right|}{\left|J_{k}\right|} \leqslant 4
$$

We call the family $\left\{I_{j}\right\}_{j}$ a Whitney decomposition of $D$. Here we use the construction in [8, pA-34]. We remark that an examination of the construction reveals that if $\left\{I_{j}^{0}\right\}_{j}$ is a Whitney decomposition of the set $D^{0} \supseteq D$, then if $I_{j} \cap I_{k}^{0} \neq \emptyset$ we have $I_{j} \subseteq I_{k}^{0}$.

Lemma 3.3 Suppose $v$ is a solution to (3.1), $b>0$ and $\phi$ is such that $b\left\|\phi^{\prime}\right\|_{L^{\infty}(\mathbb{R})}<1$.

Let I be a cube of the Whitney decomposition of $\left\{x \mid N_{2 \phi, 2 b}(v)(x)>\mu / 24\right\}$ and let $E_{\mu, \rho}$ be the intersection of an interval I with

$$
\left\{x \in \mathbb{R} \mid N_{2 \phi, b / 12}(v)(x)>\mu \text { and } C_{\phi}\left(m_{\alpha}(f)\right)(x)+S_{p, \phi, b}(v)(x) \leqslant \rho \mu\right\} .
$$


There exists a sufficiently small choice of $\rho$, independent of $I$, so that, for each $x \in E_{\mu, \rho}$, there is a interval $J$ with $x \in 6 J$ and $J \subset 3 I$ and for which

$$
\left|v\left(z, h_{2 \phi, \mu, b / 12}(v)(z)\right)\right|>\mu / 2
$$

for all $z \in J$.

Proof. See $[10,3.14]$. Let $x \in E_{\mu, \rho}$ therefore, by definition,

$$
h_{2 \phi, \mu, b / 12}(v)(x)>2 \phi(x),
$$

and so we know there exists an $\left(x_{0}, t_{0}\right)$ on $\partial \Gamma_{b / 12}\left(x, h_{2 \phi, \mu, b / 12}(v)(x)\right)$ such that $\left|v\left(x_{0}, t_{0}\right)\right|=\mu$ and we have $h_{2 \phi, \mu, b / 12}(v)\left(x_{0}\right)=t_{0}$. Set

$r_{0}=t_{0}-2 \phi(x)>0$ and $K=\left\{(z, r)|| z-x_{0}\left|\leqslant 11 b r_{0} / 72,\right| r-t_{0} \mid \leqslant r_{0} / 6\right\}$,

so then $3 K \subset \Gamma_{b}(x, 2 \phi(x))$. Moreover, since $I$ is a Whitney cube, property (b) implies that $b r_{0} \leqslant 60|I| / 33$.

Now by $[7$, Thm 8.17$]$ we have that

$$
\operatorname{OSc}_{K}(v) \leqslant C\left(r_{0}^{-1}\|v-c\|_{L^{p}(2 K)}+r_{0}^{1-2 / q}\|F\|_{L^{q}(2 K)}\right),
$$

for any constant $c$. But since $|F(z, r)| \leqslant C r_{0}^{-1} C_{\phi}\left(m_{\alpha}(f)\right)(x)$ for $(z, r) \in 2 K$,

$$
r_{0}^{1-2 / q}\|F\|_{L^{q}(2 K)} \leqslant C C_{\phi}\left(m_{\alpha}(f)\right)(x)
$$

and so, using Poincaré's inequality and (3.8),

$$
\begin{aligned}
\left|v(z, r)-v\left(x_{0}, t_{0}\right)\right| & \leqslant \operatorname{osc}_{K}(v) \leqslant C\left(\left\|\nabla(v)^{p / 2}\right\|_{L^{2}(2 K)}^{\frac{2}{p}}+C_{\phi}\left(m_{\alpha}(f)\right)(x)\right) \\
& \leqslant C\left(S_{p, \phi, b}(v)(x)+C_{\phi}\left(m_{\alpha}(f)\right)(x)\right) \leqslant C \rho \mu
\end{aligned}
$$

for any $(z, r) \in K$. Thus, we may choose $\rho$ sufficiently small, so that $\mid v(z, r)-$ $v\left(x_{0}, t_{0}\right) \mid \leqslant \mu / 2$. Then, clearly, $\left|v\left(z, h_{2 \varphi, \mu, b / 12}(v)(z)\right)\right| \geqslant \mu / 2$ for $\left|z-x_{0}\right| \leqslant$ $b r_{0} / 72$ and the lemma is proved.

Theorem 3.4 Let $p=2^{k} \geqslant 2$, for some $k \in \mathbb{N}$, $v$ be a solution to (3.1) and $\left\{I_{j}\right\}_{j}$ be the Whitney decomposition of $\left\{x \mid N_{2 \phi, 2 b}(v)(x)>\mu / 24\right\}$. Fix an interval $J$ and set $F_{j}$ equal to the intersection of $I_{j}$ with

$$
\begin{aligned}
& \left\{x \mid N_{2 \phi, b / 12}(v)(x)>\mu,\left(M_{3 J}\left(C_{\phi}\left(m_{\alpha}(f)\right)^{p}\right)<\rho \mu\right\}\right. \\
& \left.\cap\left\{x \mid M_{3 J}\left(S_{p, \phi, b}(v)^{p}\right)\right)(x)^{\frac{1}{p}}<\rho \mu\right\} \\
& \cap\left\{x \mid\left[M_{3 J}\left(N_{\phi, a}(v)^{p}\right)(x)\right]^{\frac{p-1}{p^{2}}}\left[M_{3 J}\left(C_{\phi}\left(m_{\alpha}(f)\right)^{p}\right)(x)\right]^{\frac{1}{p^{2}}}<\rho \mu\right\} \\
& \cap\left\{x \mid\left[M_{3 J}\left(N_{\phi, a}(v)^{p}\right)(x)\right]^{\frac{1}{2 p}}\left[M_{3 J}\left(S_{p, \phi, a}(v)^{p}\right)(x)\right]^{\frac{1}{2 p}}<\rho \mu\right\},
\end{aligned}
$$


where $M_{3 J}$ is the Hardy-Littlewood maximal function applied to functions restricted to $3 J$, that is $M_{3 J}(f)=M\left(\chi_{3 J} f\right)$ where $\chi_{3 J}$ is the characteristic function of $3 \mathrm{~J}$. Given $b>0$ and a Lipschitz function $\phi \geqslant 0$ such that $b\left\|\phi^{\prime}\right\|_{L^{\infty}(\mathbb{R})}<1$, there exist sufficiently small $a, \alpha$ and $\rho<1$, and a constant $c(\rho)$, independent of $j$, such that, for all $\mu>0$,

$$
\left|F_{j}\right| \leqslant c(\rho)\left|I_{j}\right|
$$

provided $I_{j} \subseteq J$. Moreover, $c(\rho)$ tends to zero as $\rho \rightarrow 0$.

Proof. Fix $j$, set $I:=I_{j}$ and $F:=F_{j}$. Define the interval

$$
I^{*}=\left\{\left(x, h_{2 \phi, \mu, b / 12}(v)(x)\right) \mid x \in I\right\} .
$$

By Lemma 3.3, for each $x \in F$ and sufficiently small $\rho$, we have that $M_{\mu}\left(v \chi_{I^{*}}\right)(x)>\mu / 12$, where $M_{\mu}$ is the Hardy-Littlewood maximal function on the graph of the function $h_{2 \phi, \mu, b / 12}(v)$. By (b), there exists a point $q$ such that $\operatorname{dist}(I, q) \leqslant 4|I|$ and $N_{2 \phi, 2 b}(v)(q) \leqslant \mu / 24$. Therefore we can choose $X_{r}$ as in Lemma 3.1, where we will take $\phi_{+}=h_{2 \phi, \mu, b / 12}(v)$, such that $\left|v\left(X_{r}\right)\right| \leqslant \mu / 24$. We have

$$
M_{\mu}\left(\left(v-v\left(X_{r}\right)\right) \chi_{I^{*}}\right)(x) \geqslant M_{\mu}\left(v \chi_{I^{*}}\right)(x)-\left|v\left(X_{r}\right)\right|>\frac{\mu}{24}
$$

for all $x \in I$, and applying the weak-type estimate for the maximal function, we obtain

$$
|F| \leqslant \frac{C}{\mu^{p}} \int_{I^{*}}\left(v-v\left(X_{r}\right)\right)^{p} .
$$

Now we can apply Lemma 3.1 to the solution $v-v\left(X_{r}\right)$, with $\phi_{+}=$ $h_{2 \phi, \mu, b / 12}(v)$, provided $a$ and $\alpha$ are sufficiently small. Observe that since we obviously have $|I|^{1 / p}\left|v\left(X_{r}\right)\right| \leqslant C\left\|N_{\phi, a}(v)\right\|_{L^{p}(3 I)}$, we get the bound

$$
\begin{aligned}
\mu^{p}|F| \leqslant & C\left(\left\|S_{p, \phi, a}(v)\right\|_{L^{p}(3 I)}^{p}+\left\|C_{\phi}\left(m_{\alpha}(f)\right)\right\|_{L^{p}(3 I)}^{p}\right. \\
& +\left\|N_{\phi, a}(v)\right\|_{L^{p}(3 I)}^{p / 2}\left\|S_{p, \phi, a}(v)\right\|_{L^{p}(3 I)}^{p / 2}+\left\|N_{\phi, a}(v)\right\|_{L^{p}(3 I)}^{p-1}\left\|C_{\phi}\left(m_{\alpha}(f)\right)\right\|_{L^{p}(3 I)} \\
& \left.+\left\|N_{\phi, a}(v)\right\|_{L^{p}(3 I)}^{(p-2) / 2}\left\|S_{p, \phi, a}(v)\right\|_{L^{p}(3 I)}^{p / 2}\left\|C_{\phi}\left(m_{\alpha}(f)\right)\right\|_{L^{p}(3 I)}\right) \\
\leqslant & C|I|\left(M_{3 J}\left(S_{p, \phi, b}(v)^{p}\right)(x)+M_{3 J}\left(C_{\phi}\left(m_{\alpha}(f)\right)^{p}\right)(x)\right. \\
& +\left[M_{3 J}\left(N_{\phi, a}(v)^{p}\right)(x)\right]^{\frac{p-1}{p}}\left[M_{3 J}\left(C_{\phi}\left(m_{\alpha}(f)\right)^{p}\right)(x)\right]^{\frac{1}{p}} \\
& \left.+\left[M_{3 J}\left(N_{\phi, a}(v)^{p}\right)(x)\right]^{\frac{1}{2}}\left[M_{3 J}\left(S_{p, \phi, a}(v)^{p}\right)(x)\right]^{\frac{1}{2}}\right) \\
\leqslant & C \rho^{p} \mu^{p}|I|
\end{aligned}
$$

and so the proof is complete. 
Corollary 3.5 Let $v$ be a solution to (3.1), $\left\{I_{k}^{0}\right\}_{k}$ be the Whitney decomposition of $\left\{x \mid N_{2 \phi, 2 b}(v)(x)>\mu_{0} / 24\right\}$ and define the set

$$
G_{k}^{0}=\left\{x \mid N_{2 \phi, b / 12}(v)(x)>\mu_{0}\right\} \cap I_{k}^{0} .
$$

For each $q>p=2^{k} \geqslant 2(k \in \mathbb{N})$, Lipschitz function $\phi \geqslant 0$ and $b>0$ such that $b\left\|\phi^{\prime}\right\|_{L^{\infty}(\mathbb{R})}<1$, there exist constants $C(p, q)$ and $C(\varepsilon, p, q)$ and sufficiently small $a$ and $\alpha$ such that

$$
\begin{aligned}
& \left\|N_{2 \phi, b / 12}(v)\right\|_{L^{q}\left(G_{k}^{0}\right)} \leqslant C(\varepsilon, p, q)\left(\left\|S_{p, \phi, b}(v)\right\|_{L^{q}\left(3 I_{k}^{0}\right)}\right. \\
& \left.\quad+\left\|C_{\phi}\left(m_{\alpha}(f)\right)\right\|_{L^{q}\left(3 I_{k}^{0}\right)}\right)+2 \varepsilon\left(\left\|N_{2 \phi, 2 b}(v)\right\|_{L^{q}\left(3 I_{k}^{0}\right)}+\left\|N_{\phi, a}(v)\right\|_{L^{q}\left(3 I_{k}^{0}\right)}\right),
\end{aligned}
$$

Proof. Fix $k$. Let $\left\{I_{j}\right\}_{j}$ and $\left\{F_{j}\right\}_{j}$ be as in Theorem 3.4 with $J=I_{k}^{0}$ and let $\left\{I_{j}^{0}\right\}_{j}$ and $\left\{F_{j}^{0}\right\}_{j}$ be the same, but with $\mu$ replaced by $\mu_{0}$. As we remarked just above Lemma 3.3, if $\mu \geqslant \mu_{0}$ and $I_{k}^{0} \cap I_{j} \neq \emptyset$, then $I_{j} \subseteq I_{k}^{0}$. This means, using Theorem 3.4, we have, for $\mu \geqslant \mu_{0}$,

$$
\begin{aligned}
\left|G_{k}^{0} \cap\left(\cup_{j} F_{j}\right)\right| & \leqslant \sum_{j: I_{k}^{0} \cap I_{j} \neq \emptyset}\left|F_{j}\right| \leqslant c(\rho) \sum_{j: I_{k}^{0} \cap I_{j} \neq \emptyset}\left|I_{j}\right| \\
& \leqslant c(\rho)\left|I_{k}^{0} \cap\left(\cup_{j} I_{j}\right)\right|
\end{aligned}
$$

Now observe if $\mu<\mu_{0}$, then $G_{k}^{0} \cap\left(\cup_{j} F_{j}\right) \subseteq F_{k}^{0}$ and $I_{k}^{0} \subseteq \cup_{j} I_{j}$. As a result of this, when $\mu<\mu_{0}$,

$$
\left|G_{k}^{0} \cap\left(\cup_{j} F_{j}\right)\right| \leqslant\left|F_{k}^{0}\right| \leqslant c(\rho)\left|I_{k}^{0}\right| \leqslant c(\rho)\left|I_{k}^{0} \cap\left(\cup_{j} I_{j}\right)\right| .
$$

Now we can proceed by standard arguments. We have

$$
\begin{aligned}
& \left\|N_{2 \phi, b / 12}(v)\right\|_{L^{q}\left(G_{k}^{0}\right)}=\int_{0}^{\infty} q \mu^{q-1}\left|\left\{x \in G_{k}^{0} \mid N_{2 \phi, b / 12}(v)(x)>\mu\right\}\right| d \mu \\
& =\int_{0}^{\infty} q \mu^{q-1}\left|G_{k}^{0} \cap\left(\cup_{j} F_{j}\right)\right| d \mu+C(\varepsilon)\left\|\left(M_{3 I_{k}^{0}}\left(S_{p, \phi, b}(v)^{p}\right)\right)^{1 / p}\right\|_{L^{q}\left(I_{k}^{0}\right)} \\
& \left.\quad+C(\varepsilon)\left\|\left(M_{3 I_{k}^{0}}\left(C_{\phi}\left(m_{\alpha}(f)\right)^{p}\right)\right)^{1 / p}\right\|_{L^{q}\left(I_{k}^{0}\right)}+\varepsilon\left\|\left(M_{3 I_{k}^{0}}\left(N_{\phi, a}(v)\right)^{p}\right)^{1 / p}\right\|_{L^{q}\left(I_{k}^{0}\right)}\right) .
\end{aligned}
$$

Therefore, using (3.10) and (3.11), this is majorised by

$$
\begin{aligned}
& c(\rho) \int_{0}^{\infty} q \mu^{q-1}\left|I_{k}^{0} \cap\left(\cup_{j} I_{j}\right)\right| d \mu+C(\varepsilon)\left\|S_{p, \phi, b}(v)\right\|_{L^{q}\left(3 I_{k}^{0}\right)} \\
& \left.+C(\varepsilon)\left\|C_{\phi}\left(m_{\alpha}(f)\right)\right\|_{L^{q}\left(3 I_{k}^{0}\right)}+\varepsilon \| N_{\phi, a}(v)\right) \|_{L^{q}\left(3 I_{k}^{0}\right)}= \\
& \left.=c(\rho) \| N_{\phi, 2 b}(v)\right)\left\|_{L^{q}\left(I_{k}^{0}\right)}+C(\varepsilon)\right\| S_{p, \phi, b}(v) \|_{L^{q}\left(3 I_{k}^{0}\right)} \\
& \left.\quad+C(\varepsilon)\left\|C_{\phi}\left(m_{\alpha}(f)\right)\right\|_{L^{q}\left(3 I_{k}^{0}\right)}+\varepsilon \| N_{\phi, a}(v)\right) \|_{L^{q}\left(3 I_{k}^{0}\right)},
\end{aligned}
$$

where we have used the boundedness of the Hardy-Littlewood maximal operator. Now choosing $\rho$ so that $c(\rho) \leqslant \varepsilon$ completes the proof of the corollary. 
Theorem 3.6 Let $v$ be a solution to (3.1). For each $l>0$, there exist constants $b, c$ and $C(p, l)$ such that

$$
\left\|N_{0, b / 12}(v)\right\|_{L^{l}(\mathbb{R})} \leqslant C(p, l)\left(\left\|S_{p, 0, c}(v)\right\|_{L^{l}(\mathbb{R})}+\left\|C_{0}(f)\right\|_{L^{l}(\mathbb{R})}\right)
$$

Proof. Fix $b$ and choose $c \geqslant 2 b$, and $a$ and $\alpha$ sufficiently small so that we may apply Corollary 3.5 with $\left\|\phi^{\prime}\right\|_{L^{\infty}(\mathbb{R})} \leqslant 1 / c$. It suffices to prove the distributional inequality

$$
\left|E_{\mu}\right| \leqslant\left(C(\varepsilon, \rho)+C \varepsilon k^{q}\right)\left|\left\{x \mid N_{0,2 b}(v)(x)>\mu / 24\right\}\right|,
$$

where

$$
\begin{array}{r}
E_{\mu}=\left\{x \mid N_{0, b / 12}(v)(x)>\mu, N_{0, c}(v)(x) \leqslant k \mu\right\} \\
\cap\left\{x \mid S_{p, 0, c}(v)(x)+C_{0}(f)(x) \leqslant \rho \mu\right\},
\end{array}
$$

$q>p$, and $C(\varepsilon, \rho)$ tends to zero as $\rho \rightarrow 0$, for each fixed $\varepsilon>0$. Let $\phi$ be the Lipschitz function for which $\partial D$ is the graph of $2 \phi$, where

$$
D=\cup_{x \in E_{\mu}} \Gamma_{c / 2}(x, 0)
$$

is a sawtooth region above $E_{\mu}$. Now let $\left\{I_{j}\right\}_{j}$ be the Whitney decomposition of $\left\{x \mid N_{2 \phi, 2 b}(v)(x)>\mu / 24\right\}$ and define the set

$$
G_{j}=\left\{x \mid N_{2 \phi, b / 12}(v)(x)>\mu\right\} \cap I_{j} .
$$

When $x \in E_{\mu}, \phi(x)=0$, and so $E_{\mu}=\cup_{j}\left(G_{j} \cap E_{\mu}\right)$. Now, for $q>p$, by Corollary 3.5,

$$
\begin{aligned}
\mu^{p} \mid G_{j} & \cap E_{\mu} \mid \leqslant C \int_{G_{j}} N_{2 \phi, b / 12}(v)^{q} \\
& \leqslant C(\varepsilon) \int_{3 I_{j}}\left(S_{p, \phi, b}(v)^{q}+C_{\phi}\left(m_{\alpha}(f)\right)^{q}\right)+2 \varepsilon \int_{3 I_{j}}\left(N_{2 \phi, 2 b}(v)^{q}+N_{\phi, a}(v)^{q}\right) \\
& \leqslant\left(C(\varepsilon) \rho^{q} \mu^{q}+C \varepsilon k^{q} \mu^{q}\right)\left|I_{j}\right|,
\end{aligned}
$$

since, as $D$ is a sawtooth domain, for any $y \in \operatorname{supp}(\phi), S_{p, \phi, b}(v)(y) \leqslant$ $S_{p, 0, c}(v)(x), N_{2 \phi, 2 b}(v)(y) \leqslant N_{0, c}(v)(x)$ and $N_{\phi, a}(v)(y) \leqslant N_{0, c}(v)(x)$ for some $x \in E_{\mu}$. Similarly, we have $C_{\phi}\left(m_{\alpha}(f)\right)(y) \leqslant C C_{0}(f)(x)$ for some $x \in E_{\mu}$.

Summing in $j$ gives

$$
\begin{aligned}
\left|E_{\mu}\right| & \leqslant\left(C(\varepsilon, \rho)+C \varepsilon k^{q}\right)\left|\left\{x \mid N_{2 \phi, 2 b}(v)(x)>\mu / 24\right\}\right| \\
& \leqslant\left(C(\varepsilon, \rho)+C \varepsilon k^{q}\right)\left|\left\{x \mid N_{0,2 b}(v)(x)>\mu / 24\right\}\right|,
\end{aligned}
$$

which concludes the proof of (3.12). 
We can now obtain the theorem using (3.12) and standard arguments. We have

$$
\begin{aligned}
& \left\|N_{0, b / 12}(v)\right\|_{L^{l}(\mathbb{R})}^{l}=\int_{0}^{\infty} l \mu^{l-1}\left|\left\{N_{0, b / 12}(v)(x)>\mu\right\}\right| d \mu \\
& \leqslant \int_{0}^{\infty} 4^{-l} l \mu^{l-1}\left(\left|E_{\mu}\right|+\left|\left\{S_{p, 0, c}(v)(x)+C_{0}(f)(x)>\rho \mu\right\}\right|\right. \\
& \left.\quad+\left|\left\{N_{0, c}(v)(x)>k \mu\right\}\right|\right) d \mu \\
& \leqslant \int_{0}^{\infty} 4^{-l} l \mu^{l-1}\left(\left(C(\varepsilon, \rho)+C \varepsilon k^{q}\right)\left|\left\{x \mid N_{0,2 b}(v)(x)>\mu / 24\right\}\right|\right. \\
& \left.\quad+\left|\left\{S_{p, 0, c}(v)(x)+C_{0}(f)(x)>\rho \mu\right\}\right|+\left|\left\{N_{0, c}(v)(x)>k \mu\right\}\right|\right) d \mu \\
& \leqslant\left(C(\varepsilon, \rho)+C \varepsilon k^{q}\right)\left\|N_{0,2 b}(v)\right\|_{L^{l}(\mathbb{R})}^{l} \\
& \quad+\frac{4^{l}}{\rho^{l}}\left(\left\|S_{p, 0, c}(v)\right\|_{L^{l}(\mathbb{R})}+\left\|C_{0}(f)\right\|_{L^{l}(\mathbb{R})}\right)+\frac{4^{l}}{k^{l}}\left\|N_{0, c}(v)\right\|_{L^{l}(\mathbb{R})}^{l} \\
& \leqslant\left(C(\varepsilon, \rho)+C \varepsilon k^{q}\right)\left\|N_{0, b / 12}(v)\right\|_{L^{l}(\mathbb{R})}^{l} \\
& \quad+\frac{4^{l}}{\rho^{l}}\left(\left\|S_{p, 0, c}(v)\right\|_{L^{l}(\mathbb{R})}+\left\|C_{0}(f)\right\|_{L^{l}(\mathbb{R})}\right)+\frac{C}{k^{l}}\left\|N_{0, b / 12}(v)\right\|_{L^{l}(\mathbb{R})}^{l},
\end{aligned}
$$

where the last inequality follows from [15, p62]. Then we may choose first $k$ sufficiently large, secondly $\varepsilon$ sufficiently small, then $\rho$ sufficiently small so that we may hide the non-tangential maximal function on the left-hand side.

It will now be useful to introduce a truncated square function, which we define as

$$
S_{p, \phi, a}^{\tau}(g)(y)=\left(\iint_{\Gamma_{a}^{\tau}(y, \phi(y))}|\nabla g(x, t)|^{2}|g(x, t)|^{p-2} d x d t\right)^{\frac{1}{p}},
$$

where $\Gamma_{a}^{\tau}(y, \phi(y))=\{(x, t)|| x-y \mid \leqslant a(t-\phi(y)), t-\phi(y) \leqslant \tau\}$ is the cone truncated at height $\tau$.

Lemma 3.7 Let $v$ be a solution to (3.1) and let $\phi$ and $\phi_{+}$be non-negative Lipschitz functions such that $\phi_{+} \geqslant 2 \phi$. Let $I$ be an interval in $\mathbb{R}$ and $r:=|I|$. Then there exists a sufficiently small choice of a and $\alpha$, depending only on $\lambda, \Lambda, p, c_{1}, \tau$ and $\left\|\phi_{+}^{\prime}\right\|_{L^{\infty}(\mathbb{R})}$, and a constant $C(p)$, depending on the same parameters, along with $a$ and $\alpha$, such that

$$
\begin{aligned}
\left\|S_{p, \phi, a}^{\tau r}(v)\right\|_{L^{p}((1 / 2) I)}^{p} \leqslant & C(p)\left(\left\|N_{\phi, a}(v)\right\|_{L^{p}(3 I)}^{p}\right. \\
& +\left\|N_{\phi, a}(v)\right\|_{L^{p}(3 I)}^{p-1}\left\|C_{\phi}\left(m_{\alpha}(f)\right)\right\|_{L^{p}(3 I)} \\
& +\left\|N_{\phi, a}(v)\right\|_{L^{p}(3 I)}^{(p-2) / 2}\left\|S_{p, \phi, a}(v)\right\|_{L^{p}(3 I)}^{p / 2}\left\|C_{\phi}\left(m_{\alpha}(f)\right)\right\|_{L^{p}(3 I)} \\
& \left.+\left\|N_{\phi, a}(v)\right\|_{L^{p}(3 I)}^{p / 2}\left\|S_{p, \phi, a}(v)\right\|_{L^{p}(3 I)}^{p / 2}\right) .
\end{aligned}
$$


Proof. We perform the same calculation as we did in the proof of Lemma 3.1, but with $\xi_{2}$ replaced by $\xi_{2}(\tau \cdot)$. The second term on the right-hand side of (3.7) can be controlled by $\left\|N_{\phi, a}(v)\right\|_{L^{p}(3 I)}^{p}$. Then, with the same notation as Lemma 3.1, we see $\left\|f_{0} \xi_{1}\right\|_{L^{p}(\mathbb{R})}^{p}=\mathrm{I}+\mathrm{II}+\mathrm{III}+\mathrm{IV}$. The first term of $\mathrm{II}_{2}$ in (3.9) can be combined with I to produce

$$
\iint_{\mathbb{R}_{+}^{2}} \nabla w \cdot A \nabla w \xi \frac{2 t d x d t}{a_{22}} \geqslant C\left\|S_{p, 0, a}^{\tau r}(v)\right\|_{\left.L^{p}((1 / 2) I)\right)}^{p} .
$$

and all the other terms can be bounded about as before to obtain the theorem.

Theorem 3.8 Let $p \geqslant 2, v$ be a solution to (3.1) and $\left\{I_{j}\right\}_{j}$ be the Whitney decomposition of $\left\{x \mid S_{p, \phi, b}(v)(x)>\mu / 4\right\}$. Fix an interval $J$ and set

$$
\begin{aligned}
F_{j}=I_{j} & \cap\left(\left\{x \mid S_{p, \phi, a}(v)(x)>\mu\right\}\right. \\
& \cap\left\{x \mid\left(M_{6 J}\left(C_{\phi}\left(m_{\alpha}(f)\right)^{p}\right)+M_{6 J}\left(N_{\phi, b}(v)^{p}\right)\right)(x)^{\frac{1}{p}}<\rho \mu\right\} \\
& \cap\left\{x \mid\left[M_{6 J}\left(N_{\phi, a}(v)^{p}\right)(x)\right]^{\frac{p-1}{p^{2}}}\left[M_{6 J}\left(C_{\phi}\left(m_{\alpha}(f)\right)^{p}\right)(x)\right]^{\frac{1}{p^{2}}}<\rho \mu\right\} \\
& \cap\left\{x \mid\left[M_{6 J}\left(N_{\phi, a}(v)^{p}\right)(x)\right]^{\frac{1}{2 p}}\left[M_{6 J}\left(S_{p, \phi, a}(v)^{p}\right)(x)\right]^{\frac{1}{2 p}}<\rho \mu\right\} \\
& \left.\cap\left\{x \mid\left[M_{6 J}\left(C_{\phi}\left(m_{\alpha}(f)\right)^{p}\right)(x)\right]^{\frac{1}{2 p}}\left[M_{6 J}\left(S_{p, \phi, a}(v)^{p}\right)(x)\right]^{\frac{1}{2 p}}<\rho \mu\right\}\right),
\end{aligned}
$$

where $M_{6 J}$ is the Hardy-Littlewood maximal function applied to functions restricted to $6 J$, that is $M_{6 J}(f)=M\left(\chi_{6 J} f\right)$ where $\chi_{6 J}$ is the characteristic function of $6 J$. Given a Lipschitz function $\phi \geqslant 0$ such that $b\left\|\phi^{\prime}\right\|_{L^{\infty}(\mathbb{R})}<1$ and for sufficiently small $a, \alpha$ and $\rho<1$, there exists a constant $c(\rho)$, independent of $j$, such that for all $\mu>0$

$$
\left|F_{j}\right| \leqslant c(\rho)\left|I_{j}\right|
$$

provided $I_{j} \subseteq J$. Moreover, $c(\rho)$ tends to zero as $\rho \rightarrow 0$.

Proof. Fix $j$ and set $I:=I_{j}$ and $F:=F_{j}$. By property (b) of the Whitney decomposition we know that if $x \in F$, then there exists a point $x^{\prime} \in 8 I$ such that $S_{\phi, b}(v)\left(x^{\prime}\right) \leqslant \mu / 4$. Therefore, since we may assume $b>a$,

$$
S_{p, \phi, a}^{\tau r}(v)(x) \geqslant S_{p, \phi, a}(v)(x)-S_{p, \phi, b}(v)\left(x^{\prime}\right) \geqslant \mu-\mu / 4>\mu / 2,
$$

for some $\tau>0$, depending only on $a, b$ and $\left\|\phi^{\prime}\right\|_{L^{\infty}(\mathbb{R})}$. Thus,

$$
\mu^{p}|F| \leqslant C \int_{I} S_{p, \phi, a}^{\tau r}(v)(x)^{p} d x
$$

and we may apply Lemma 3.7 to obtain the Theorem via standard arguments. 
Corollary 3.9 Let $q>p \geqslant 2$, $v$ be a solution to (3.1). There exists a constant $C(p, q)>0$, depending only on $\lambda, \Lambda, c_{1}, p$ and $q$, such that

$$
\left\|S_{p, 0, a}(v)\right\|_{L^{q}(\mathbb{R})} \leqslant C(p, q)\left(\left\|N_{0, a}(v)\right\|_{L^{q}(\mathbb{R})}+\left\|C_{0}(f)\right\|_{L^{q}(\mathbb{R})}\right)
$$

Lemma 3.10 Let $v$ be a solution to (2.4) (that is, (3.1) with $f_{0} \equiv 0$ ). Then there exists a constant $c_{2}$, depending only on $a, p$ and the ellipticity constants, such that

$$
\left\|S_{p, 0, a}(v)\right\|_{L^{p}(\mathbb{R})} \leqslant C\left(p, c_{1}\right)\left\|C_{0}(f)\right\|_{L^{p}(\mathbb{R})},
$$

for some $C\left(p, c_{1}\right)>0$, depending on the same parameters and $c_{1}$, provided $c_{1}<c_{2}$.

Proof. We perform the same calculation as we did in the proof of Lemma 3.1, but with $\xi \equiv 1$. With the same notation, but with $w$ replaced by $v$ (as we have no need of the transformation $\Phi)$, we see $\left\|f_{0}\right\|_{L^{p}(\mathbb{R})}^{p}=\mathrm{I}+\mathrm{II}+\mathrm{IV}$. The first term of $\mathrm{II}_{2}$ in (3.9) can be combined with I to produce

$$
\iint_{\mathbb{R}_{+}^{2}} \nabla v \cdot A \nabla v \frac{2 t d x d t}{a_{22}} \geqslant C\left\|S_{p, 0, a}(v)\right\|_{L^{p}(\mathbb{R})}^{p} .
$$

and all other terms can be bounded about as before. We can then use Cauchy's Inequality and Theorem 3.6 to hide $\left\|N_{0, a}(v)\right\|_{L^{p}(\mathbb{R})}$ and $\left\|S_{p, 0, a}(v)\right\|_{L^{p}(\mathbb{R})}$ as necessary. The only terms for which this is not possible are $\mathrm{II}_{3}$ and the third term on the right-hand side of (3.9), so it is here we use that $c_{1}$ is small.

Lemma 3.11 For be a solution $v$ to (3.1) and its conjugate $\bar{v}$, we have

$$
\left\|S_{2,0, a}(v)\right\|_{L^{p}\left(\partial \mathbb{R}_{+}^{2}\right)} \leqslant C(p)\left(\left\|S_{2,0, a}(\bar{v})\right\|_{L^{p}\left(\partial \mathbb{R}_{+}^{2}\right)}+\left\|C_{0}(f)\right\|_{L^{p}\left(\partial \mathbb{R}_{+}^{2}\right)}\right)
$$

for $1<p<\infty$.

Proof. Using (2.5) and (2.3) it is easy to see

$$
S_{2,0, a}(v)(x)^{2} \leqslant C\left(S_{2,0, a}(\bar{v})(x)^{2}+\iint_{\Gamma_{a}(x, 0)}|F|^{2}\right)
$$

so, to complete the proof, it suffices to show

$$
\int\left(\iint_{\Gamma_{a}(x, 0)}|F|^{2}\right)^{\frac{p}{2}} d x \leqslant C\left\|C_{0}(f)\right\|_{p}^{p} .
$$


We can do this via duality [1], testing $F$ against $g$ such that

$$
x \mapsto\left(\iint_{\Gamma_{a}(x, 0)}|G(y, s)|^{2} \frac{d y d s}{s^{2}}\right)^{\frac{1}{2}} \in L^{p^{\prime}}(\mathbb{R}),
$$

where $\frac{1}{p}+\frac{1}{p^{\prime}}=1$. Indeed,

$$
\begin{aligned}
\iint_{\mathbb{R}_{+}^{2}} G(x, t) F(x, t) d x d t & \leqslant \iint_{\mathbb{R}_{+}^{2}} m_{\alpha}(G)(x, t) f(x, t) d x d t \\
& \leqslant \int_{\mathbb{R}} N_{0, a / 2}\left(m_{\alpha}(G)\right)(x) C_{0}(f)(x, t) d x d t \\
& \leqslant\left\|N_{0, a / 2}\left(m_{\alpha}(G)\right)\right\|_{L^{p^{\prime}(\mathbb{R})}}\left\|C_{0}(f)\right\|_{L^{p}(\mathbb{R})} .
\end{aligned}
$$

But since

$$
N_{0, a / 2}\left(m_{\alpha}(G)\right)(x) \leqslant\left(\iint_{\Gamma_{a}(x, 0)}|G(y, s)|^{2} \frac{d y d s}{s^{2}}\right)^{\frac{1}{2}},
$$

the proof of (3.13) is complete.

\section{References}

[1] Coifman, R. R., Meyer, Y. And Stein, E. M.: Some new function spaces and their applications to harmonic analysis. J. Funct. Anal. 62 (1985), no. $2,304-335$.

[2] Dahlberg, B. E. J.: On the Poisson integral for Lipschitz and $C^{1}$ domains. Studia Math. 66 (1979), no. 1, 13-24.

[3] Dahlberg, B. E. J.: Approximation of harmonic functions. Ann. Inst. Fourier (Grenoble) 30 (1980), no. 2, 97-107.

[4] Dahlberg, B. E. J.: Weighted norm inequalities for the Lusin area integral and the nontangential maximal functions for functions harmonic in a Lipschitz domain. Sudia Math. 67 (1980), no. 3, 297-314.

[5] Dahlberg, B. E. J., Kenig, C. E., Pipher, J. and Verchota, G. C.: Area integral estimates for higher order elliptic equations and systems. Ann. Inst. Fourier (Grenoble) 47 (1997), no. 5, 1425-1461.

[6] Dindoš, M., Petermichl, S. And Pipher, J.: The $L^{p}$ Dirichlet problem for second order elliptic operators and a $p$-adapted square function. J. Funct. Anal. 249 (2007), no. 2, 372-392.

[7] Gilbarg, D. and Trudinger, N. S.: Elliptic Partial Differential Equations of Second Order. Springer-Verlag, Heidelberg, 1997.

[8] Grafakos, L.: Classical and Modern Fourier Analysis. Pearson Education, Upper Saddle River, NJ, 2004. 
[9] Jerison, D. S. And Kenig, C. E.: The Neumann problem on Lipschitz domains. Bull. Amer. Math. Soc. 4 (1981), no. 2, 203-207.

[10] Kenig, C. E., Koch, H., Pipher, J. and Toro, T.: A new approach to absolute continuity of elliptic measure, with applications to non-symmetric equations. Adv. Math. 153 (2000), no. 2, 231-298.

[11] Kenig, C. E. And Pipher, J.: The Dirichlet problem for elliptic equations with drift terms. Publ. Mat. 45 (2001), no. 1, 199-217.

[12] Kenig, C. E. And Rule, D. J.: The regularity and Neumann problem for non-symmetric elliptic operators. Trans. Amer. Math. Soc. 361 (2009), no. $1,125-160$.

[13] NeČAs, J.: Les méthodes directes en théorie des équations elliptiques. Masson et Cie, Éditeurs, Paris; Academia, Éditeurs, Prague, 1967.

[14] Pipher, J.: Littlewood-Paley estimates: Some applications to elliptic boundary value problems. In Partial differential equations and their applications (Toronto, ON, 1995), 221-238. CRM Proc. Lecture Notes 12. Amer. Math. Soc., Providence, RI, 1997.

[15] Stein, E. M.: Harmonic Analysis: Real-Variable Methods, Orthogonality and Oscillatory Integrals. Princeton Mathematical Series 43. Princeton University Press, Princeton, NJ, 1993.

Recibido: 17 de febrero de 2009

Martin Dindoš

School of Mathematics and Maxwell Institute for Mathematical Sciences University of Edinburgh King's Buildings Mayfield Road, Edinburgh, EH9 3JZ, UK m.dindos@ed.ac.uk

David J. Rule

Dept. of Mathematics and Maxwell Institute for Mathematical Sciences

Colin Maclaurin Building Heriot-Watt University Edinburgh, EH14 4AS, UK rule@uchicago.edu 